\title{
激光去除与连接技术发展研究
}

\author{
陈俐，巩水利，何恩光 \\ （中国航空制造技术研究院高能束流加工技术重点实验室，北京 100024）
}

\begin{abstract}
摘要：随着激光技术的发展，激光去除与连接技术深刻影响着制造业技术的发展，并以柔性、高效、高质的综合优势成为不 可或缺的先进制造技术。在先进制造智能化发展的时代，激光技术、数字信息技术等的融合必将推动激光去除与连接技术的 应用发展与创新。本文选取工业应用最广的激光焊接、激光切割、激光打孔、激光打标 4 种激光去除与连接技术，概述了激 光去除与连接技术的应用现状，分析了其在支撑先进制造发展过程中存在的问题。研究表明，激光去除与连接技术的发展应 注重工艺基础和装备研究一体化的研究, 解决激光加工单元部件、激光加工工艺、智能集成各环节的关键技术, 强化工艺基 础研究平台建设, 关注技术创新和人才培养的多维度结合, 以促进激光去除与连接技术从工艺应用开发到装备市场保障的生 态链式的协同发展。
\end{abstract}

关键词: 激光加工; 激光焊接; 激光切割; 激光打孔；激光打标

中图分类号: U664.5 文献标识码: A

\section{Development of Laser Cutting and Joining Processing}

\section{Chen Li, Gong Shuili, He Enguang}

(Key Laboratory of Science and Technology on Power Beam Processes, AVIC Manufacturing Technology Institute, Beijing 100024, China)

\begin{abstract}
Laser cutting and joining technology is indispensable as an advanced manufacturing technology owing to its comprehensive advantages of flexibility, high efficiency, and high quality; and it has a profound impact on the manufacturing industry. With the intelligentization of advanced manufacturing, the laser cutting and joining technology will be surely promoted by the integration of laser technology and digital manufacturing technology. This study selects and analyzes four specific technologies that are most widely used in industry to summarize the application status of the laser joining and cutting technology, namely, laser welding, laser cutting, laser drilling, and laser marking. The problems faced by the development of the laser joining and cutting technology are discussed. The study shows that the development of laser cutting and joining technology should focus on the integration of the fundamental processes and equipment so as to improve key technologies in laser processing unit, laser materials processing, and intelligent integration. Moreover, research platforms for fundamental processes should be strengthened, with a focus on multi-dimensional combination of technological innovation and talent training. This will promote the coordinated development of the laser cutting and joining technology from application development to equipment market guarantee.
\end{abstract}

Keywords: laser material processing; laser welding; laser cutting; laser drilling; laser marking

收稿日期 : 2020-03-12; 修回日期 : 2020-05-08

通讯作者：陈俐, 中国航空制造技术研究院高能束流加工技术重点实验室研究员, 研究方向为激光加工技术; E-mail: ouchenxi@163.com 资助项目：中国工程院咨询项目“我国激光技术与应用 2035 发展战略研究” (2018-XZ-27)

本刊网址： www.engineering.org.cn/ch/journal/sscae 


\section{一、前言}

自 1960 年第一台红宝石激光器问世以来, 激 光技术已从 $\mathrm{CO}_{2}$ 激光、固体激光（YAG）时代跨越 到光纤激光、碟片激光和半导体激光时代。激光技 术不断驱动着先进制造技术的变革，在结构轻量化 设计制造、绿色制造等方面发挥越来越重要的作用。 其中, 以激光切割、激光焊接、激光打孔、激光打 标为主体的激光去除与连接技术是最为广泛和活跃 的激光加工应用技术，已在航空、航天、车辆、舰 船和工程机械等工业领域得到了广泛应用 [1 3]。据 1996-2016 年全球激光加工技术专利数据分析 [4], 激光焊接、激光切割和激光打孔是激光加工技术创 新发展的重点, 这也从侧面反映出激光去除与连接 技术的庞大市场需求。

激光可在能量域、时间域、频域和空间域进行多 维度的调控，有利于实现高效、高精、高质量制造， 对产品设计和制造工艺观念的创新起着促进作用。而 将激光加工工艺及装备与计算机数控技术、传感与信 息传输技术、智能化技术结合，将更有利于激光在制 造领域展现其提高产品质量和劳动生产率的优势。世 界先进国家或地区均已将激光加工列为先进制造技术 发展方向，我国也已将激光加工技术定位为国家建设 的关键支撑技术。智能制造成为全球制造业变革的主 要方向 [5], 激光去除与连接技术作为一种重要的制造
技术也不例外。激光加工工艺及装备如何与数字化、 智能化深度融合, 是激光加工制造领域面向 2035 年 迫切需解决的问题，也是当今制造业转型升级和制造 技术水平提升发展的关键。

本文针对激光去除与连接技术，在总结其技术 内涵的基础上，梳理当前技术的发展现状与存在问 题，分析其关键技术情况，并结合应用需求提出推 动激光去除与连接技术发展的对策建议。

\section{二、激光去除与连接技术概述}

激光去除与连接技术的本质是利用激光束与材料 间的热力耦合效应, 诱导材料产生热化学变化, 使零 件或构件材料实现标记、刻蚀、去除、固化或熔化连 接, 包括激光焊接、激光切割、激光打孔、激光打标 等多种工艺, 一般而言, 激光去除与连接技术的具体 工艺实施与激光热效应相关, 取决激光功率密度和激 光作用时间的匹配关系，如图 1 所示。

激光去除与连接技术因采用的激光类型和加工 工作原理的不同，已衍生出多种多样的工艺技术(见 图 2)。激光去除与连接技术可采用不同波长的激 光, 目前主要有 $\mathrm{CO}_{2}$ 激光、YAG 激光、光纤激光 和半导体激光; 可采用连续激光加工和脉冲激光加 工, 如毫秒激光、微秒激光、纳秒激光以及皮秒、 飞秒超快激光等脉冲激光; 当激光器特性不同时,

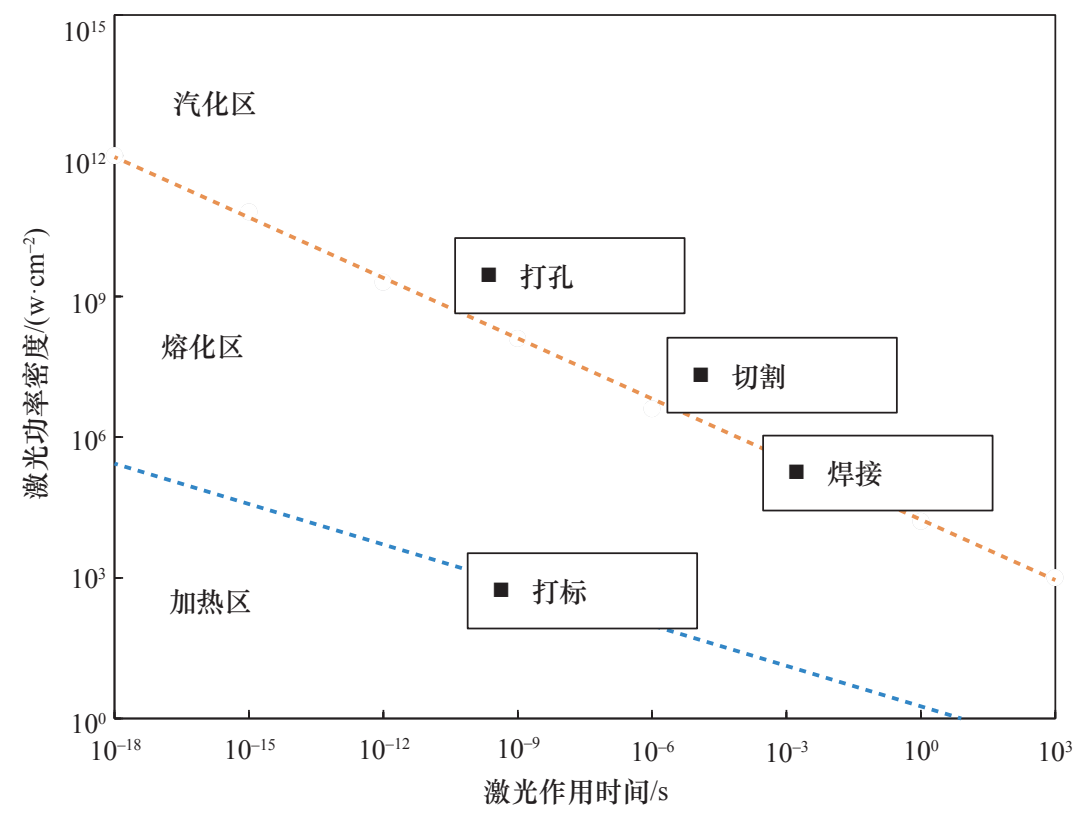

图 1 激光功率密度与激光作用时间对热效应的影响 


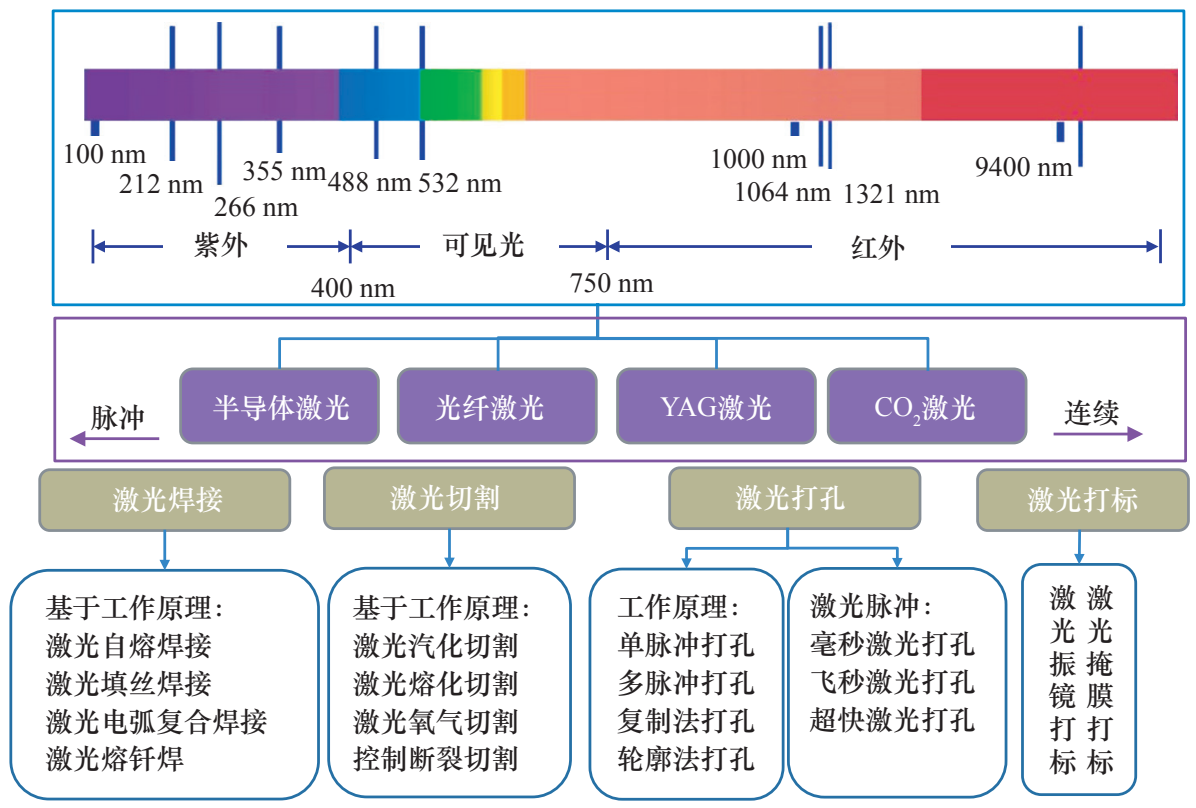

图 2 激光去除与连接技术分类

激光束流运行控制方式不同，工作原理也不同，以 适应对不同材料、不同结构的加工。

\section{(一) 激光焊接}

激光焊接利用可聚焦调控激光束使材料熔化、 汽化形成熔池, 熔池凝固结晶形成冶金连接。与传 统焊接方法相比, 激光焊接加热集中、焊接变形小, 在提高结构材料利用率、减轻结构重量、降低成本 方面具有显著优势。激光焊接主要有激光热导焊接 和激光深熔焊接两种方式，其中，激光热导焊接主 要应用于仪器仪表、电子元件等微结构的精密焊接, 激光深熔焊主要应用于汽车、舰船、航空、航天、 核电等领域的大尺度结构焊接。为解决光斑直径细 小导致的焊接装配精度要求高和高反射性材料激光 焊接困难等问题, 激光焊接衍生出多种工艺, 如采 用复合工艺形式的激光填丝焊接、激光电弧复合焊 接和激光熔钎焊接等, 采用光束控制的多光斑激光 焊接、振镜激光焊接等。

\section{（二）激光切割}

激光切割利用激光使材料熔化、汽化, 辅助 高速气流去除熔融物质而实现材料分离或去除。激 光切割具有切割热影响区小、质量好、效率高、变 形小的优势, 且可切割范围广泛, 如各种金属和包 括玻璃、木材、皮革等的非金属。激光切割可分为
4 类工艺: 激光汽化切割, 多用于金属和非金属薄 材, 需大功率密度激光; 激光熔化切割, 主要用于 不锈钢、钛、铝及其合金等; 激光氧气切割, 主要 用于碳钢等易氧化金属, 以激光和氧气氧化反应热 为能量, 切割速度远远大于激光汽化切割和熔化切 割; 激光控制断裂切割, 主要用于热脆性材料, 利 用材料热梯度形成裂缝而快速切断。另外, 激光切 割的工艺控制参数主要有激光功率、切割速度、焦 点位置及辅助气体, 其工艺及装备的研发也主要围 绕这些参数展开 [6]。

\section{（三）激光打孔}

激光打孔是利用高能脉冲激光束去除材料加工 孔的方法, 不受材料硬度、刚性、强度和脆性等性 能限制, 而且速度快、效率高。激光打孔需要光、 机、电一体化技术的协同，可实现对微细孔、斜孔、 不规则孔及密集群孔的加工, 如发动机单晶叶片气 膜孔 [7]。其中, 光束旋转是最常见的激光打孔方 法, 特点是孔壁质量好、速度快、可多孔加工。光 束直接穿孔适合孔径 0.05 0.6 $\mathrm{mm}$ 的微孔加工, 切 割打孔适合大尺度、多孔加工，激光束旋转适合于 孔径 0.05 3 $\mathrm{mm}$ 的单孔加工。

\section{（四）激光打标}

激光打标利用激光对材料局部进行照射, 使其 
表面汽化刻蚀或发生化学反应而形成永久标记, 具 有对零件无损伤、标记清晰的优势。激光打孔适用 于多种材料, 如集成电子模块、汽车玻璃、医疗器 械、橡胶制品、手机面板等, 还可适用于聚合物光 纤表面功能性制备 [8]。激光打标可利用激光掩膜 扫描实现, 也可通过多台激光器发射脉冲阵列实现, 且光束扫描可机械驱动, 也可振镜驱动。

\section{三、激光去除与连接技术发展现状}

激光去除与连接技术是激光在工业领域的定 制应用。该技术在欧洲、美国等发达国家或地区已 构建了从激光器源开发到激光加工装备研制的良好 产业生态链，使激光去除与连接技术渗透到制造业 的各个领域。经过十余年的发展, 我国在激光去除 与连接技术方面的应用研发已在国际上占有一席之 地, 激光产业链也具备一定基础, 但在高端装备方 面与国际水平相比还存在差距。随着互联网时代的 到来, 工业领域对激光设备和生产过程的智能化提 出了更高需求。

\section{（一）激光焊接}

美国、欧洲等国家和地区自 20 世纪 80 年代中 期就关注激光焊接应用。1985 年第一块激光拼焊板 应用于奥迪 100 车身; 2005 年空客 380 采用了铝合 金激光焊接机身下壁板结构, 成为航空制造技术的 标志 [3]。当前在节能减排的大背景下, 全球对激 光焊接结构的应用需求增多, 对激光焊接工艺提升、 质量保证、激光焊接装备等也提出更高要求 [9]。近 年来, 我国激光焊接技术飞速发展, 已应用于飞机 壁板、轨道客车等方面 $[3,10]$ 。目前光纤激光是激 光焊接新工艺的研究热点, 未来低成本、高功率的 半导体激光焊接以及先进激光器与高端数控技术、 机器人技术结合的焊接工艺及装备集成化将成为重 要的发展趋势。

\section{（二）激光切割}

激光切割是激光加工中应用最早、最多的技术。 我国激光切割应用日益广泛, 激光切割工艺及装备 的研发逐步由钢结构扩展为有色金属结构。与此同 时, 国外激光切割工艺及设备的研发趋于高端化,
在关注激光器优化提升、切割过程模拟仿真研究的 基础上, 加强对大功率、智能化激光切割设备和特 殊环节激光切割技术的研发 [6]。当前, 激光切割 工艺在我国传统产业升级改造过程中的应用潜力增 大, 亟需将激光切割工艺与现有技术相匹配, 推广 激光切割工艺到更多的生产领域, 并提高大功率激 光切割设备的智能化水平。

\section{（三）激光打孔}

激光打孔是工业领域备受关注的制孔技术发展 方向, 激光打孔的效率是电火花加工的 12 15 倍, 是机械钻孔的 200 倍, 并使难加工孔的加工成为可 能。在激光打孔工艺中目前最受关注的是飞秒激光 打孔工艺, 可应用于发动机气膜冷却叶片孔的制备。 与纳秒激光打孔相比, 飞秒激光打孔时会产生冷加 工效应, 可避免加工过程的再铸层和微裂纹, 同时, 通过设备智能控制系统来实现光束自适应调节与工 件高速自动定位技术的结合, 从而保证气膜孔超精 细加工 [11]。

\section{（四）激光打标}

激光打标的应用发展过程与激光打标控制系统 的发展历程基本吻合。1995-2003 年，激光打标先 后经历了大幅面时代、转镜时代和振镜时代, 与此 同时, 激光打标控制系统也经历了从直接控制到上 下位机控制, 再到实时处理、分时复用控制的发展 过程。目前, 随着半导体激光、光纤激光和紫外激 光在激光打标中的应用, 对激光打孔的过程控制提 出了新的要求。

\section{四、激光去除与连接技术发展存在的问题}

工业激光器和智能技术的不断发展, 使激光去 除与连接技术由 “激光制造” 不断向 “激光智造” 演进, 亟需激光去除与连接技术进行战略转型和核 心技术提升，并与信息技术、智能技术深度融合。 虽然近年来激光去除与连接工艺设备集成化、智能 化研制已取得了显著进展, 市场规模也逐步扩大, 但如何基于先进激光技术和高端装备技术、基于数 字化将工艺集成和设备集成融为一体 (见图 3), 切 实提升激光去除与连接核心技术，仍存在不少问题 
需解决, 具体如下。

\section{（一）工艺认知与信息化}

激光去除与连接技术的应用需加强工艺基础研 究。从新材料、新结构的角度，工艺研发是发现缺 陷如何形成、如何消除必不可少的环节; 从新工艺、 新方法的角度，工艺研发是揭示缺陷如何控制、如 何检测的关键。面向数字化技术，激光去除与连接 技术工艺研究的挑战是在材料、工艺和装备之间建 立起精细化、精量化的数字联系，打造产品全周期 制造的仿真系统，提取智能化关键要素。

\section{（二）激光束源与工艺性}

从激光微加工到大功率激光加工, 激光去除与 连接技术加工工艺多种多样。随着未来激光去除与 连接高速、高效激光加工技术的发展, 对高品质、 高稳定的激光器提出了更高要求。而我国激光器的 研发却出现了脱节, 制约了高端激光器的应用, 亟 需强化与激光束源调控相结合的工艺基础研究。

\section{（三）加工单元与工艺性}

激光去除与连接技术作为一种特种材料加工技 术，其工艺的可靠实施主要依靠激光加工单元的功 能性和可控性来实现。具体来看, 与数字化控制相
结合的光作用能量和轨迹精确控制, 结合工艺需求 激光的能量域、时间域、频域和空间域的多维度控 制, 如旋转扫描加工单元，使熔池摚拌激光焊接成 为可能，而其应用拓展更应关注如何将工艺经验移 植到机器控制, 以提高加工单元的智能性。

\section{（四）激光去除与连接设备智能集成内涵}

设备智能集成的目标是实现激光去除与连接技 术高效率、高精度制造。一方面是激光控制、运动 控制及检测系统等的集成; 另一方面是工艺优化、 自主决策工具模块的集成，而更重要的是信息流的 传输与集成, 包括基于机理研究的工艺数字仿真信 息、工艺大数据分析信息和多层面的控制数据信息, 这些信息的理论研究和工具开发将是今后支撑激光 去除与连接技术智能化发展的重要支柱。

\section{五、激光去除与连接关键技术分析}

面向 2035 年的先进制造技术，激光去除与连 接技术的发展需从工艺机理出发, 将工程应用与信 息技术深度融合，在制造过程全周期的数据信息资 源中探寻关键技术环节和流程，关注工艺设备集成 和信息集成的共性与个性问题的研发，关注激光去 除与连接技术的智能内涵, 推进关键技术的突破。

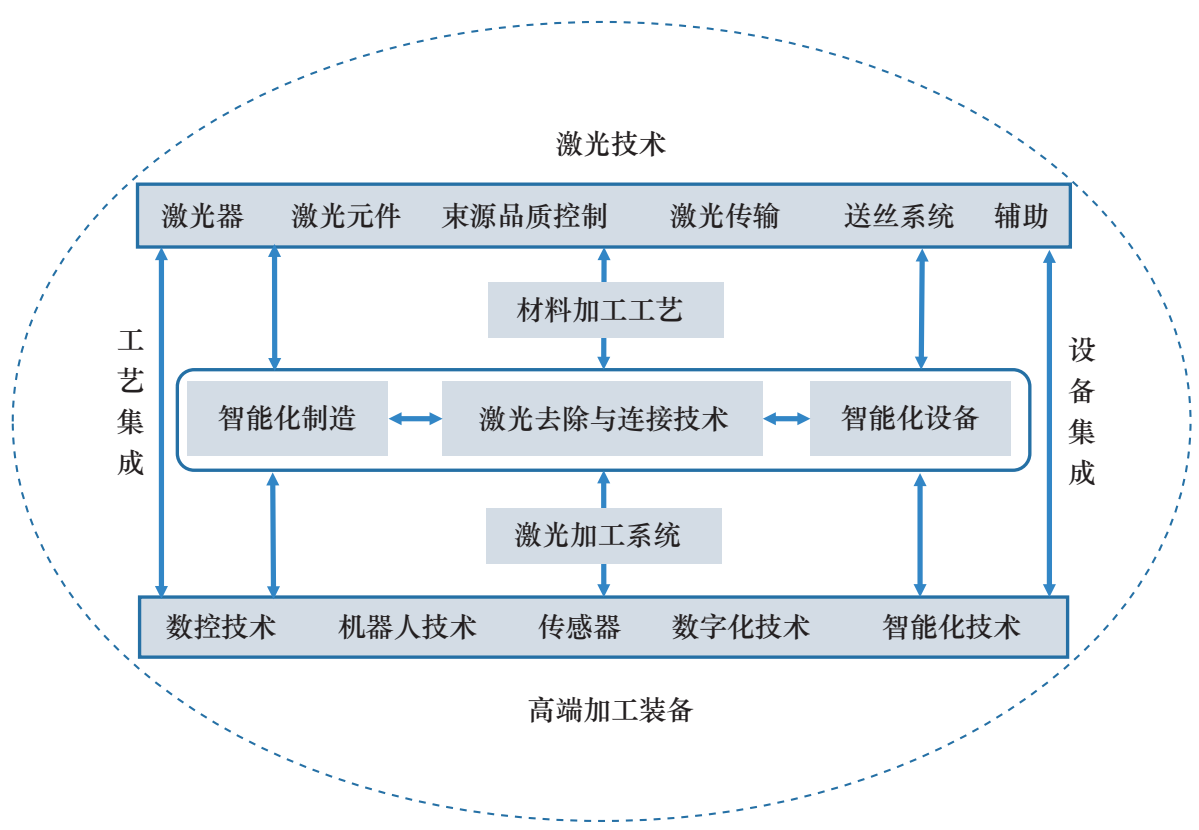

图 3 激光去除与连接技术的工艺设备集成 
就激光去除与连接技术应用拓展而言，应重点考虑 以下关键技术。

1. 基于大数据信息分析的工艺优化与规划

激光去除与连接工艺机理涉及激光与材料间复 杂热力耦合作用, 材料自身物态、性能变化与光能 量控制、光运动控制密切相关，而工艺优化目标是 建立工艺参数 / 成形质量 / 组织性能的相关性。但 当前涉及工艺基础研究的数值模拟和试验分析仍然 处于 “孤岛” 状态，工艺优化的不确定性制约着激 光去除与连接技术的智能化发展。因此，基于大数 据理念, 将揭示工艺机理科学本质的数值模拟数据 转变为工艺可控, 把试验分析数据深化提炼为科学 规律，是解决制造过程全周期工艺优化与规划的关 键，也是激光去除与连接虚拟制造的关键。

2. 激光加工单元柔性化研制技术

在激光加工集成与智能化的过程中，激光加工 单元柔性化是关键。一方面光控制和辅助参量控制 的集成, 解决了激光加工精细化的问题, 如激光焊 接丝、气、光的运动协同和参量协调; 另一方面, 激光加工单元的感应感知功能集成、数据流的传输, 解决了加工单元 “眼” 与控制系统 “脑” 的集成。 未来激光加工单元技术的发展方向是将激光束源的 调控技术融入到激光加工单元中。

\section{3. 激光加工设备智能集成化技术}

将激光加工的源、运动及检测系统等集成以完 成全自动化、数字化、智能化加工是激光去除与连 接技术发展的方向。而激光加工的智能化不是简单 的机器人、数控技术和大数据技术的应用, 而是基 于激光加工过程多维能场的交互作用的认知和感知 研究。通过光、机、电、材料、工艺、控制、信息 各学科深度融合, 以激光轨迹运动为中心多系统的 集成以及软件工具集成将起关键支撑作用。

\section{六、结语}

激光去除与连接技术智能化发展是今后的重要 发展趋势。在技术层面，不仅需关注与新一代信息 技术、数字化制造技术和激光技术的有机融合，而 且需关注激光去除与连接在产品制造全生命周期的 数字化技术应用, 而对工艺过程的科学认知是智能 化发展的基础，工艺与信息融合软件集成、工艺控 制与设备控制的集成是发展的支撑。
激光去除与连接技术的发展依赖多学科融合推 进，政府引导规划，多方参与共建是发展之本。首 先，搭建高水平工艺创新研究平台，建设工艺基础、 高端装备研发基地，吸纳相关企业、科学家的参与, 支撑新原理、新方法的挖掘, 培养创新性人才, 孵 化激光去除与连接技术的新产品。其次，政企联合 研发布局, “从源到果” 聚焦激光器、激光加工单 元和新工艺的技术研发，关注满足产品生命全周期 制造的重点项目立项，通过增强高端技术开发能力、 平衡中端技术建设能力、调控低端技术的发展布局， 有序引导激光去除与连接市场生态的发展。

\section{参考文献}

[1] 左铁钏, 陈虹. 21世纪的绿色制造一一激光制造技术及应用 [J]. 机械工程学报, 2009, 45(10): 106-110.

Zuo T C, Chen H. Green manufacture in 21 century-Laser manufacturing technology and application $[\mathrm{J}]$. Journal of Mechanical Engineering, 2009, 45(10): 106-110.

[2] 肖荣诗, 陈铠, 陈涛. 激光制造技术的现状及发展趋势 [J]. 电加 工与模具, 2009 (S1): 18-22.

Xiao R S, Chen K, Chen T. Review of laser advanced manufacturing technology [J]. Electro-machining \& Mould, 2009 (S1): $18-22$.

[3] 巩水利. 先进激光加工技术 [M]. 北京: 航空工业出版社, 2016. Gong S L. Advanced laser materials processing technology [M]. Beijing: Aviation Industry Press, 2016

[4] 赵蕴华, 高铭, 周立娟. 基于专利分析的全球激光加工技术创新 趋势研究 [J]. 高技术通讯, 2017, 27(8): 769-775.

Zhao Y H, Gao M, Zhou L J. A study of the trends of laser processing technology innovation based on patent analysis $[\mathrm{J}]$ High Technology Letters, 2017, 27(8): 769-775.

[5] 中国科协智能制造学会联合体. 中国智能制造重点领域发展报 告2018 [M]. 北京: 机械工业出版社, 2019.

Intelligent Manufacturing Alliance of CAST Member Societies. 2018 development report on key field of intelligent manufacturing in China [M]. Beijing: China Machine Press, 2019.

[6] 孙晓东, 王松, 赵凯华, 等. 激光切割技术国内外研究现状 [J]. 金 属铸锻焊技术, 2012, 41(9): 214-216.

Sun X D, Wang S, Zhao K H, et al. Research progress of laser cutting technology domestic and overseas [J]. Hot Working Technology, 2012, 41(9): 214-216.

[7] 张文武, 郭春海, 张天润, 等. 浴轮叶片先进气膜冷却与相关激 光打孔技术进展 [J]. 航空制造技术, 2016, 59(22): 26-31.

Zhang W W, Guo C H, Zhang T R, et al. Advanced film cooling technology of turbine blades and progress in relevant laser drilling technology [J]. Aeronautical Manufacturing Technology, 2016, 59(22): 26-31.

[8] 漆宇, 刘楚嘉, 何涌, 等. 利用激光打标法制备侧面均匀发光聚 合物光纤 [J]. 光学学报, 2018, 38(12): 44-53.

Qi Y, Liu C J , He Y, et al. Uniform side-glowing polymer optical fiber fabricated by laser-marking [J]. Acta Optica Sinica, 2018 , 
38(12): 44-53.

[9] 张妍. 激光焊接在白车身上的应用现状 [J]. 电焊机, 2016, 46(3): 122-126.

Zhang Y. Application status of laser welding technology in BIW [J]. Electric Welding Machine, 2016, 46(3): 122-126.

[10] 韩晓辉, 张志毅, 李刚卿. 激光焊接技术在不锈钢轨道客车制造 中的应用与展望 [J]. 电焊机, 2018, 48(3): 1-8.
Han X H, Zhang Z Y, Li G Q. Application and prospect of laser welding technology in manufacturing of the stainless steel railway vehicles [J]. Electric Welding Machine, 2018, 48(3): 1-8.

[11] 孙黎. 气膜冷却孔飞秒激光加工技术与设备. [J]. 航空动力, 2018 (5): 61-64.

Sun L. Femtosecond laser machining technologies and equipments for film cooling holes [J]. Aerospace Power, 2018 (5): 61-64. 